\title{
Effect of Huangqin Tang on Urine Metabolic Profile in Rats with Ulcerative Colitis Based on UPLC-Q-Exactive Orbitrap MS
}

\author{
Dunfang Wang, ${ }^{1}$ Xuran Ma, ${ }^{1}$ Shanshan Guo, ${ }^{1}$ Yanli Wang, ${ }^{1}$ Tao Li, $^{2}$ Dixin Zou, ${ }^{1}$ \\ Hongxin Song, ${ }^{1}$ Weipeng Yang $\mathbb{D}^{1},{ }^{1}$ and Yongxiang Ge $\mathbb{D}^{3}$ \\ ${ }^{1}$ Institute of Chinese Materia Medica, China Academy of Chinese Medical Sciences, Beijing 100700, China \\ ${ }^{2}$ The Experimental Research Center, China Academy of Chinese Medical Sciences, Beijing 100700, China \\ ${ }^{3}$ Beijing Hospital of Integrated Traditional Chinese and Western Medicine, Beijing 100039, China
}

Correspondence should be addressed to Weipeng Yang; wpyang@icmm.ac.cn and Yongxiang Ge; gyx19722007@163.com

Received 19 August 2019; Revised 12 February 2020; Accepted 13 February 2020; Published 22 April 2020

Academic Editor: Yoshiyuki Kimura

Copyright (c) 2020 Dunfang Wang et al. This is an open access article distributed under the Creative Commons Attribution License, which permits unrestricted use, distribution, and reproduction in any medium, provided the original work is properly cited.

\begin{abstract}
As a classic prescription, Huangqin Tang (HQT) has been widely applied to treat ulcerative colitis (UC), although its pharmacological mechanisms are not clear. In this study, urine metabolomics was first analysed to explore the therapeutic mechanisms of HQT in UC rats induced by TNBS. We identified 28 potential biomarkers affected by HQT that might cause changes in urine metabolism in UC rats, mapped the network of metabolic pathways, and revealed how HQT affects metabolism of UC rats. The results showed that UC affects amino acid metabolism and biosynthesis of unsaturated fatty acids and impairs the tricarboxylic acid cycle (TCA cycle). UC induced inflammatory and gastrointestinal reactions by inhibiting the transport of fatty acids and disrupting amino acid metabolism. HQT plays key roles via regulating the level of biomarkers in the metabolism of amino acids, lipids, and so on, normalizing metabolic disorders. In addition, histopathology and other bioinformatics analysis further confirm that HQT altered UC rat physiology and pathology, ultimately affecting metabolic function of UC rats.
\end{abstract}

\section{Introduction}

Ulcerative colitis (UC) is a common inflammatory bowel disease, with abdominal pain, diarrhea, mucus, and pus, sustained or repeated as the main symptom [1]. The disease is considered to be a precancerous lesion of colon cancer, and it has been listed as one of the refractory diseases by the World Health Organization. It is widely believed that UC is the result of a combination of factors including genetics, gastrointestinal flora imbalance, and inflammatory overreaction [2-4]. Traditional Chinese medicine (TCM) is a complex and interactive system, which is frequently used in the form of formula (the combination of several different herbs) [5]. Compared with chemical treatment, TCM has the advantage of fewer adverse effects and is increasingly attracting researchers' attention for the treatment of gastrointestinal diseases [6]. Huangqin Tang (HQT), a wellknown classic prescription for curing diarrhea, is a combination of four herbal medicines $3: 2: 2: 2$ by weight, namely, Scutellaria baicalensis Georgi, Paeonia lactiflora Pall, Glycyrrhiza uralensis Fisch, and Ziziphus jujuba Mill. Our previous work found that it has a good effect on intestinal mucosal inflammation in UC rat models $[7,8]$.

Metabolomics is defined as systematically qualitative and quantitative analysis of metabolites in a given organism or biological sample [9], which together with genomics, transcriptomics, and proteomics jointly constitutes the "Systems Biology" [10, 11]. As a systemic approach, metabolomics reflects the function of organisms from terminal symptoms of metabolic network by a "top-down" strategy and shows metabolic changes of a complete system caused by interventions in a holistic context $[12,13]$. As an emerging field, LC-MS-based metabolomics has been frequently applied to the toxicity study of pure compounds, extracts, and compound prescriptions and is a good tool to evaluate toxicity of natural products systematically and 
explore the mechanisms of toxicities. Now, the focus of metabolic toxicity research is mainly on nephrotoxicity, hepatotoxicity, cardiotoxicity, and central nervous system toxicity [11].

UPLC-based metabonomics was also employed to analyse the key endogenous metabolites in the body fluids, which is now increasingly considered as a novel diagnostic approach in clinical studies including liver, lung, gastrointestinal, diabetes, urogenital, and other diseases. Simultaneously, novel and more sensitive biomarkers were found for early detection and diagnosis of these diseases [14-16]. The clinical application of metabonomics provides comprehensive information and improves the feasibility of highthroughput patient screening for diagnosis of disease status or risk evaluation. Most likely, identification of clinically relevant metabolites that may be regarded as potential new biomarkers will also help with the evaluation of prognosis and contribute to the development of new therapeutic strategies [17].

Through literature search, there are few reports on the metabolomics research of UPLC-MS combined with UC. Therefore, this study used metabolomics UPLC-MS technology to find biomarkers related to UC. At the same time, the effects of HQT on the metabolites of UC rats were analyzed, and the possible metabolic pathways in rats were revealed to further elucidate the mechanism of HQT in the treatment of UC.

\section{Materials and Methods}

2.1. Chemicals. 2,4,6-Trinitrobenzenesulfonic acid was obtained from Sigma-Aldrich (St. Louis, MO, USA). IL-17 and $\mathrm{PGE}_{2}$ ELISA kits were purchased from Shanghai Panke Industrial Co., Ltd (Shanghai, China). The RNA PCR Kit was sourced from Takara (Dalian, China). Formic acid (LC-MS grade) was purchased from Tedia Company Inc. (Fairfield, $\mathrm{OH}, \mathrm{USA}$ ). Acetonitrile and methanol (LC-MS grade) were purchased from Sigma-Aldrich.

2.2. Animals. Wistar male rats (180-200 g) were obtained from the Laboratory Animal Center of the Academy of Military Medical Sciences (Production license no. SCXK 2012-0004). All rats were housed at $23 \pm 1.5^{\circ} \mathrm{C}$. Animal experiment process was conducted in accordance with the ethical guidelines for local animal care and usage.

2.3. Preparations of HQT. Scutellaria baicalensis Georgi, Paeonia lactiflora Pall, Glycyrrhiza uralensis Fisch, and Ziziphus jujuba Mill (weight ratio $3: 2: 2: 3$ ) were weighed and mixed. For the first decoction, the mixture was refluxed with water $(1: 10, \mathrm{w} / \mathrm{v})$ for $1.5 \mathrm{~h}$. The filtrates were collected, and then the water with residues $(1: 8, \mathrm{w} / \mathrm{v})$ was boiled for an additional $1 \mathrm{~h}$. Two batches of filtrates were gathered. Thereafter, the sample was dried under reduced pressure to obtain the HQT extract. Furthermore, the major active ingredients in the prescription were detected by using liquid chromatography-tandem mass spectrometer (LC-MS).
2.4. UC Rat Model Construction and Grouping. TNBS colitis in rats was induced according to previously reported methods [18]. $24 \mathrm{~h}$ fasted Wistar rats were moderately anesthetized with pentobarbital, and then carefully inserted a $0.56 \mathrm{~mm}$ catheter into the colon with the tip $8 \mathrm{~cm}$ proximal to the anus. To break the intestinal epithelial barrier and induce colitis, the mixed reagent containing $0.25 \mathrm{ml}$ of $50 \%$ ethanol and TNBS $(100 \mathrm{mg} / \mathrm{kg})$ was slowly administered into the lumen by the catheter fitted onto a $1 \mathrm{ml}$ syringe. Rats in the control group were all administered an equal volume of saline. Animals were kept in an upside-down position for 2 min and returned to their cages.

2.5. Serum Cytokine Detection. After a ten-day administration, blood samples were collected by the eyelid method and then centrifuged $(3,000 \mathrm{rpm}, 15 \mathrm{~min})$ to get serum. Production of NO in serum was measured by Griess assay; the levels of proinflammatory cytokines IL-17 and $\mathrm{PGE}_{2}$ in serum were detected by using ELISA kits in accordance with the manufacturer's specifications.

2.6. Histological Examination. After the colonic segments were collected from all three rat groups, the tissue was embedded and sliced as usual. Then, $4 \mu \mathrm{m}$-thick tissue sections were prepared and stained with hematoxylin and eosin (H\&E) for histological studies. The extent of colonic lesions was compared based on the ulcer size, inflammatory infiltration, and structural damage.

2.7. Reverse Transcription-PCR. Colonic segments were frozen and ground in liquid nitrogen. Then, $2 \mu \mathrm{g}$ RNA was used for reverse transcription. Primers for FABP1 and FABP2 and GAPDH are given in Table 1. Moreover, the mixture was diluted 10 -fold, and final reaction volumes of $20 \mu \mathrm{l}$ were used for data analysis. Reactions were run in triplicate. The relative levels of FABP1 and FABP2 were calculated by the $2^{-\Delta \Delta \mathrm{Ct}}$ method [19].

2.8. Urine Sample Collection. After the last administration, all rats were fasted for 24 hours, and then urine was collected the next morning and centrifuged for $10 \mathrm{~min}$ at $3000 \mathrm{rpm}$. Each urine sample $(50 \mu \mathrm{L})$ was added to $450 \mu \mathrm{L}$ acetonitrile agent. The samples were vortexed for 30 seconds and centrifuged at 13,000 rpm for 10 minutes at $4^{\circ} \mathrm{C}$. Thereafter, $100 \mu \mathrm{L}$ supernatant was transferred to an autosampler vial for analysis with a UPLC-Q-Exactive hybrid quadrupoleOrbitrap mass spectrometer. The mobile phases included A $(0.1 \%$ formic acid and $2 \mathrm{mmol} / \mathrm{L}$ ammonium formate in water) and B (acetonitrile). The gradient procedure was as follows: $0 \sim 1 \mathrm{~min}, 95 \sim 95 \% \mathrm{~A} ; 1 \sim 15 \mathrm{~min}, 95 \% \sim 5 \% \mathrm{~A}$; $15 \sim 16 \mathrm{~min}, 5 \% \sim 5 \% \mathrm{~A} ; 16 \sim 17 \mathrm{~min}, 5 \% \sim 95 \% \mathrm{~A}$; and 17 $\sim 18 \mathrm{~min}, 95 \% \sim 95 \% \mathrm{~A}$. The analysis time of the sample is 0 to 18 minutes. The ion source optimal conditions were set as follows: evaporation temperature, $350^{\circ} \mathrm{C}$; sheath gas, $35 \mathrm{Arb}$; spray voltage, $2.8 \mathrm{kV}$; auxiliary gas, $10 \mathrm{Arb}$; capillary temperature, $320^{\circ} \mathrm{C}$; and S-lens RF, 50. The compound parameters were set as follows: grade I, full scan $( \pm)$; resolution, 
TABle 1: Primer used for the quantitative PCR.

\begin{tabular}{lc}
\hline Gene & \multicolumn{1}{c}{ Primer sequence $5^{\prime} \longrightarrow 3^{\prime}$} \\
\hline \multirow{2}{*}{ FABP1 } & F: $5^{\prime}$-TACCAAGTGCAGAGCCAAGAG-3' \\
& R: $5^{\prime}$-TGACCTTTTCCCCAGTCATGG-3' \\
\hline \multirow{2}{*}{ FABP2 } & F: $5^{\prime}$-TGGGCATTAACGTGGTGAAGA-3' \\
& R: $5^{\prime}$-GTCCAGGTCCCAGTGAGTTC-3' \\
\hline \multirow{2}{*}{ GAPDH } & F: $5^{\prime}$-GAGTCAACGGATTTGGTCGT-3' \\
& R: $5^{\prime}$-GACAAGCTTCCCGTTCTCAG-3' \\
\hline
\end{tabular}

70,000; maximum TT, $100 \mathrm{~ms}$; and AGC target, $1 \mathrm{e}^{6} ; \mathrm{m} / \mathrm{z}$, $70-1050$.

2.9. Statistical Analysis. The mzCloud (Thermo Fisher Scientific) was used to obtain the exact mass [20]. Then, we used TraceFinder software (Thermo Fisher Scientific) to qualitatively analyze the peak area of each endogenous metabolite in the sample. Next, identified ions were confirmed by databases including Metlin (http://metlin.scripps.edu/), the Human Metabolome Database (http://www.hmdb.ca) [21], and the Kyoto Encyclopedia of Genes and Genomes (http:// www.genome.jp/kegg/ligand.html) [22, 23]. Principal component analysis (PCA) and partial least squares-discriminant analysis (PLS-DA) were performed using MetaboAnalyst 4.0 to identify discriminant metabolites. The data were statistically processed using SPSS 11.0 (SPSS Inc, Chicago, IL, USA). Our data were expressed as mean\pm standard deviation (SD), and Student's $t$-test or one-way ANOVA was used for comparison between groups.

2.10. Metabolic Pathways Analysis. Pathway analysis of potential biomarkers (VIP values $>1$ ) was assessed using MBRole. The critical $p$ value obtained from pathway analysis was set as $<0.01$. Then, we used the Metscape model, which was utilized to visualize interactions among compounds, enzymes, and genes in Cytoscape, to map a functional network of the major endogenous metabolites related to UC.

\section{Results}

3.1. Chemical Compounds of HQT. LC-MS was performed to identify the major active ingredients in HQT. The 11 main active-ingredient chemical compositions were as follows: baicalin $(10.4149 \%)$, wogonoside $(2.4179 \%)$, oroxylin-Aglucoside $(0.8229 \%)$, baicalein $(0.6754 \%)$, wogonin $(0.2215 \%)$, oroxylin-A $(0.0967 \%)$, liquiritin $(0.0384 \%)$, isoliquiritin apioside $(0.0354 \%)$, liquiritigenin $(0.0280 \%)$, isoliquiritoside $(0.0865 \mu \mathrm{g} / \mathrm{mL})$, and isoliquiritigein $(0.0049 \%)$ [24].

3.2. HQT Promoted Recovery of the UC Rats. The UC rats induced by TNBS showed a series of symptoms within 3 days, such as apathetic, drowsiness, poor appetite, weight loss, diarrhea, and purulent stools. After prolonged HQT exposure, rats' response to abovementioned symptoms were all improved significantly. Their appetite was stimulated with decreased diarrhea. The model group rats had no obvious improvement in symptoms.

\subsection{HQT Decreases Serum Level of Inflammatory Cytokines.} The levels of proinflammatory factors NO, IL-17, and $\mathrm{PGE}_{2}$ in the model group were increased remarkably. In addition, levels of NO, IL-17, and $\mathrm{PGE}_{2}$ in the HQT group were clearly lower than those in the model group (Figure 1).

3.4. Histological Study. The rats with TNBS-induced colitis in the mucosa of colons revealed inflammatory cell infiltration, loss or enlargement of goblet cells and epithelium, and edema. Part of the colon gland disappeared. The histologic sections of the HQT group showed progressive restoration, improvement of intestinal ulcer, and reduction in infiltration of inflammatory cells and edema compared to the UC group (Figure 2).

3.5. The $m R N A$ Levels of FABP1 and FABP2. The results of real-time PCR analyses indicated that the mRNA levels of FABP1 $(P<0.05)$ and FABP2 $(P<0.01)$ in colon tissue of UC model rats were significantly lower than those of normal rats but could be effectively increased by HQT treatment (Figure 3).

3.6. Diversified Analysis of Pattern Recognition of Urine Data. Urine data from the control group and the model group were analyzed using unsupervised PCA. It can be seen from the scores in Figure 4(a) that there is a certain tendency for each group of samples to aggregate. In order to obtain more ideal intergroup separation and enhance the identification of variables that contribute to the classification, a supervised PLS-DA analysis was further carried out. As shown in Figure 4(b), the model group is clearly separated from the normal group, and the normal physiological metabolism of the rat body was disturbed. From the perspective of the change of physiological metabolites, the UC model can be considered to be successful. In Figure 4(c), there is no overlap between the normal group, the model group, and the HQT group. The HQT group is gradually closer to the normal group than the model group. It indicated that the endogenous metabolic pattern of the urinary part of UC rats changed after HQT administration, which indicated that HQT had a certain effect on regulating metabolic abnormalities in UC rats. The quality parameters of UC models were accuracy, 0.8947; $R^{2}, 0.8255$; and $Q^{2}, 0.6065$.

3.7. Identification of Potential Biomarkers. In the PLS-DA model, 28 variables (VIP $>1.0$ ) were identified as metabolic markers, as shown in Tables 2 and 3. Compared with the normal group, in the model group, L-isoleucine, pyroglutamic acid, L-histidine, 4-hydroxyproline, 3-methylhistidine, gamma-aminobutyric acid, L-valine, L-proline, L-leucine, glycine, m-methylhippuric acid, 5-hydroxyindoleacetic acid, 1,2,3-propanetricarboxylic acid, ethanolamine, adipic acid, and 4-hydroxyphenylacetic acid were 


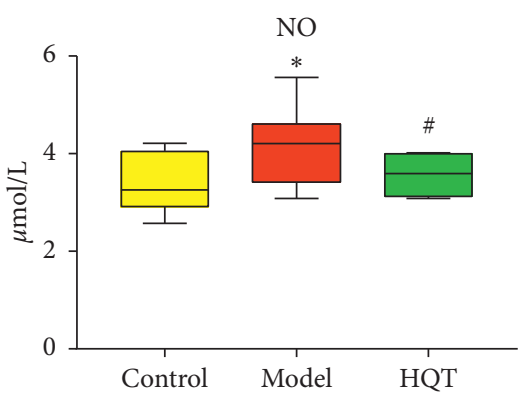

(a)

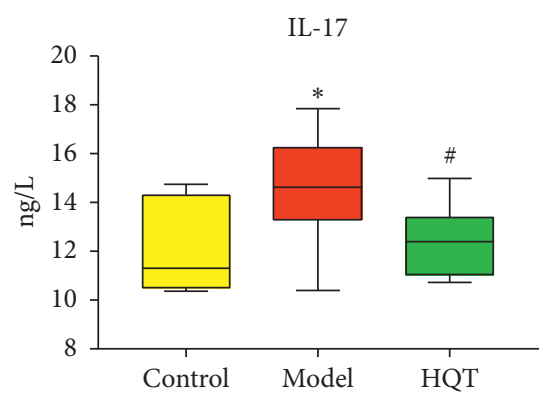

(b)

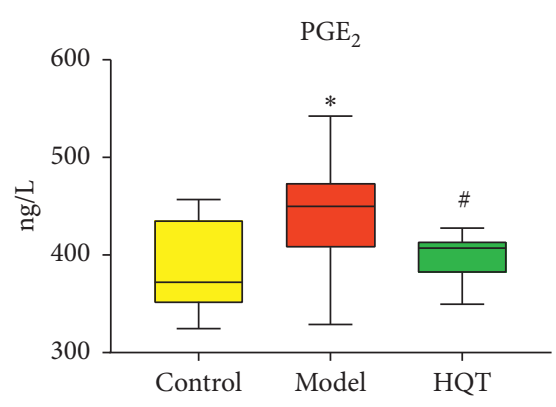

(c)

Figure 1: Effects of Huangqin Tang (HQT) on concentrations of (a) NO, (b) IL-17, and (c) PGE 2 after 10 days of oral administration on ulcerative colitis rats. $(n=10,(\bar{x}) \pm s){ }^{*} P<0.05,{ }^{* *} P<0.01$ vs normal control; ${ }^{\#} P<0.05$, ${ }^{\# \#} P<0.01$ vs model control.

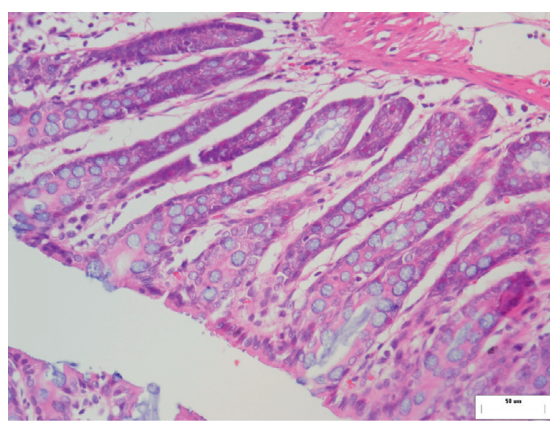

(a)

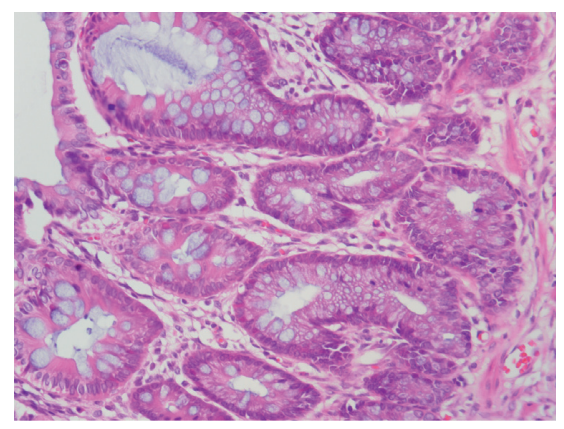

(b)

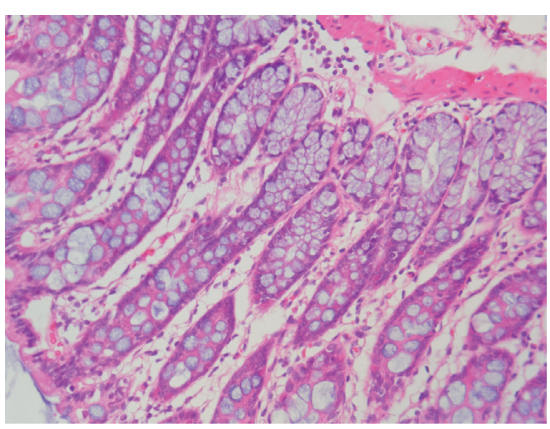

(c)

Figure 2: Histological observations of the colon tissues in different groups (H\&E staining) $(10 \times 40)$. (a) Normal control; (b) UC Model control; (c) HQT $20 \mathrm{~g} \cdot \mathrm{kg}^{-1}$.

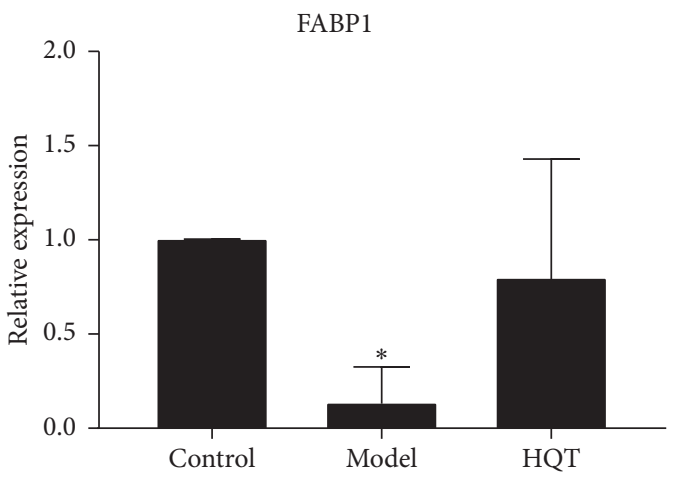

(a)

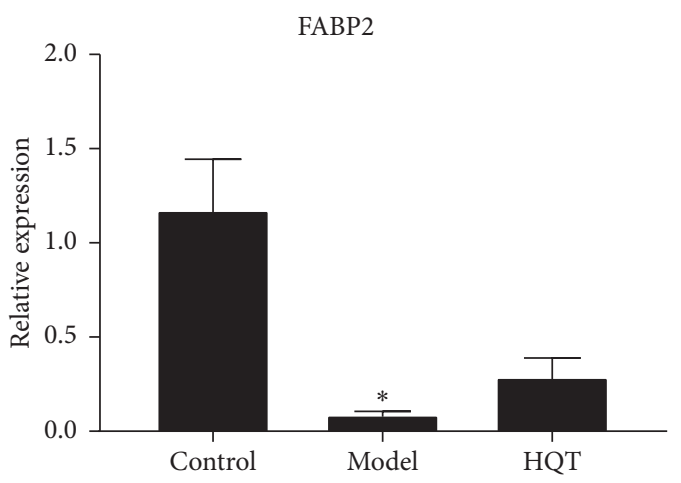

(b)

FiguRE 3: Effect of HQT on mRNA expression levels of the corresponding genes (a) FABP1 and (b) FABP2 according to real-time PCR analysis. Data are represented as mean \pm S.E. “*”, “**” and $P<0.05, P<0.01$, respectively, as compared with the normal control group. “\#” and "\#\#", $P<0.05$ and $P<0.01$, respectively, as compared with the model group.

significantly elevated; arachidonic acid, palmitoleic acid, alpha-linolenic acid, and oleic acid were significantly reduced. After the intervention of HQT, the abovementioned multiple differential metabolites have a tendency to approach normal levels, indicating that HQT has a regulating effect on the abovementioned metabolites. However, adipic acid, 4-hydroxyproline, etc., did not approach the normal value after drug treatment. The reasons for consideration may be as follows: on the one hand, the treatment time is still short; on the other hand, the disease changes still need a certain process.

3.8. Correlation and Cluster Analysis of Latent Biomarkers. The correlations between the trends of potential molecular markers are shown in the form of heat maps (Figure 5). The horizontal and vertical axes represent the variable information of endogenous substances, the light color indicates 

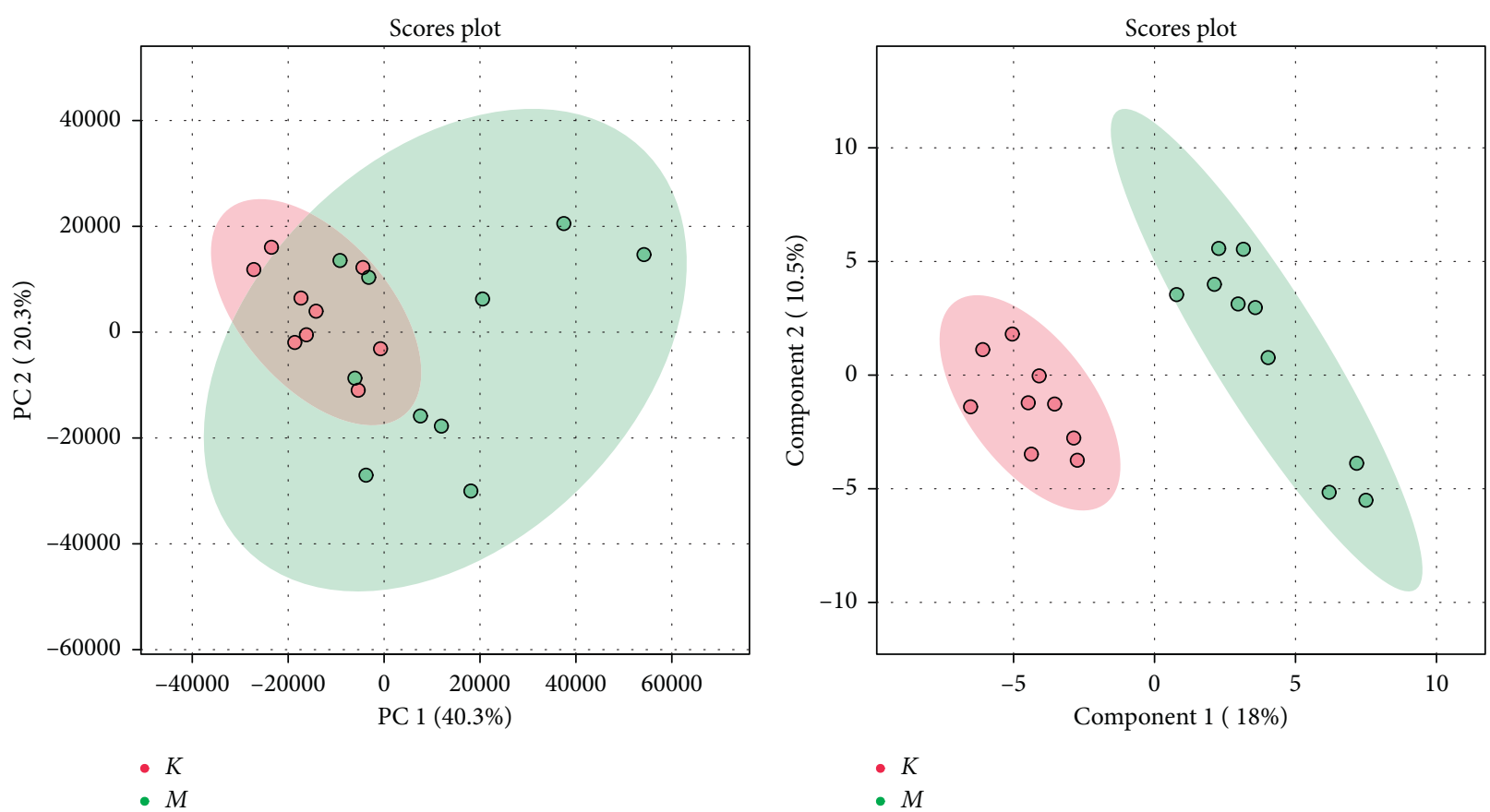

(a)

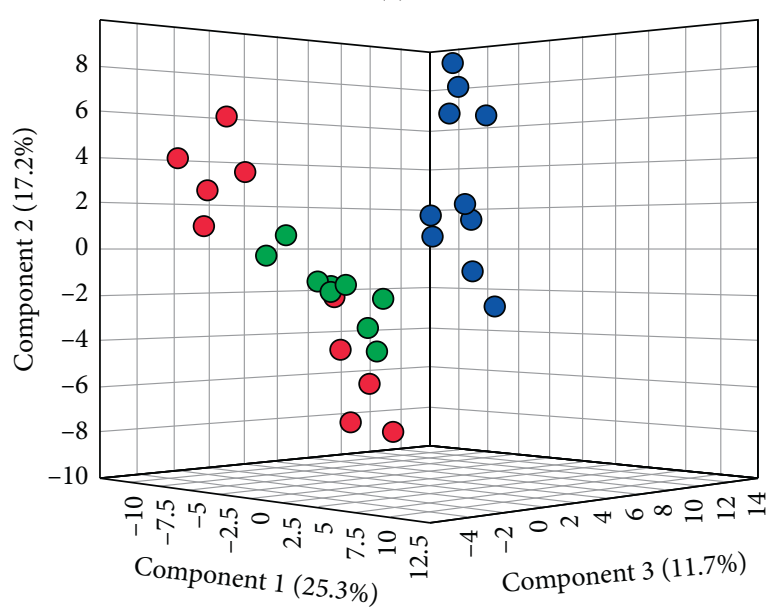

$M$

(b)

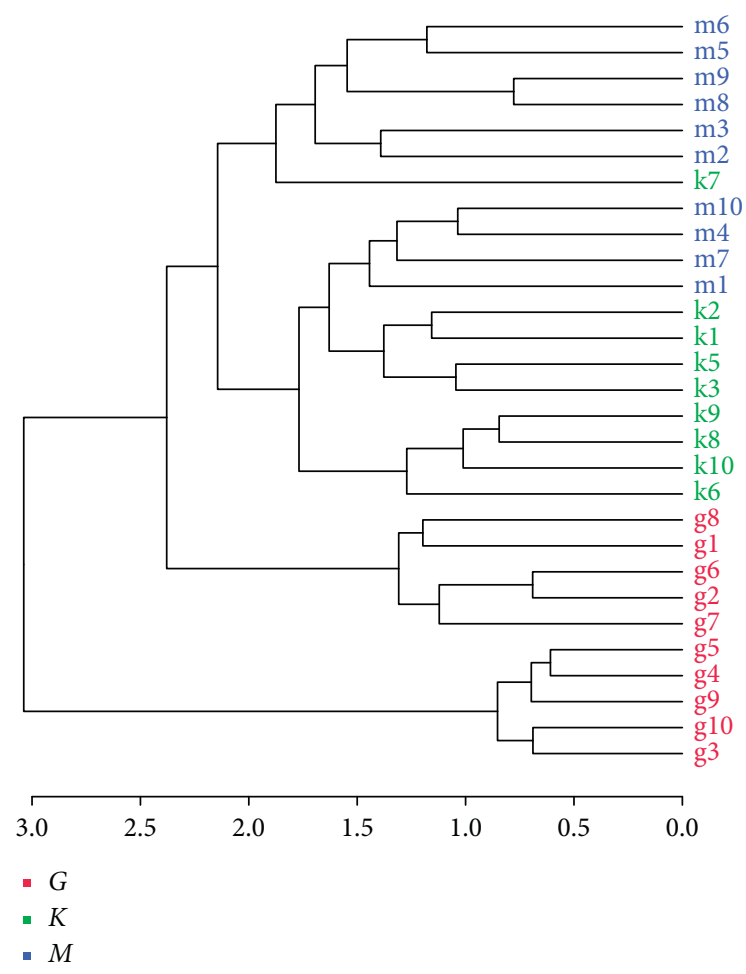

(c)

(d)

FIGURE 4: Metabolomics analysis of urine samples from control, model, and HQT rats ( $K$ denotes the control group, $M$ denotes the model group, and $G$ denotes the HQT group). (a) PCA score plots for comprehensive metabolomic data of the model and control groups. (b) PLS-DA score plots for comprehensive metabolomic data of the model and control groups. (c) Score plots of the OPLS-DA of the model, control, and HQT groups. (d) Cluster analysis was performed on the model group, control group, and HQT group (distance measurement method: euclidean; clustering method: ward. D).

weak correlations, and dark color reflects strong correlations. Blue denotes a negative correlation and red indicates a positive correlation. The closer the correlation value is to 1 , the more similar the trend between the two molecules is. Conversely, the correlation is less than zero, indicating that the change trend between the two molecular markers is 
TABLE 2: Potential biomarkers of ulcerative colitis.

\begin{tabular}{|c|c|c|c|c|}
\hline Metabolite & $\mathrm{RT}(\min )$ & Actual mass & Formula & KEGG \\
\hline m-Methylhippuric acid & 3.70 & 193.1992 & $\mathrm{C}_{10} \mathrm{H}_{11} \mathrm{NO}_{3}$ & - \\
\hline 5-Hydroxyindoleacetic acid & 3.47 & 191.1834 & $\mathrm{C}_{10} \mathrm{H}_{9} \mathrm{NO}_{3}$ & C05635 \\
\hline 1,2,3-Propanetricarboxylic acid & 0.99 & 176.1241 & $\mathrm{C}_{6} \mathrm{H}_{8} \mathrm{O}_{6}$ & C19806 \\
\hline Ethanolamine & 1.21 & 61.0831 & $\mathrm{C}_{2} \mathrm{H}_{7} \mathrm{NO}$ & C00189 \\
\hline Adipic acid & 1.05 & 146.1412 & $\mathrm{C}_{6} \mathrm{H}_{10} \mathrm{O}_{4}$ & $\mathrm{C} 06104$ \\
\hline L-isoleucine & 1.02 & 131.1729 & $\mathrm{C}_{6} \mathrm{H}_{13} \mathrm{NO}_{2}$ & C00407 \\
\hline Pyroglutamic acid & 0.87 & 129.114 & $\mathrm{C}_{5} \mathrm{H}_{7} \mathrm{NO}_{3}$ & C01879 \\
\hline L-histidine & 13.52 & 155.1546 & $\mathrm{C}_{6} \mathrm{H}_{9} \mathrm{~N}_{3} \mathrm{O}_{2}$ & C00135 \\
\hline 4-Hydroxyproline & 0.93 & 131.1299 & $\mathrm{C}_{5} \mathrm{H}_{9} \mathrm{NO}_{3}$ & C01157 \\
\hline Sarcosine & 0.87 & 89.0932 & $\mathrm{C}_{3} \mathrm{H}_{7} \mathrm{NO}_{2}$ & $\mathrm{C} 00213$ \\
\hline 3-Methylhistidine & 1.66 & 169.1811 & $\mathrm{C}_{7} \mathrm{H}_{11} \mathrm{~N}_{3} \mathrm{O}_{2}$ & $\mathrm{C} 01152$ \\
\hline Gamma-aminobutyric acid & 0.96 & 103.1198 & $\mathrm{C}_{4} \mathrm{H}_{9} \mathrm{NO}_{2}$ & $\mathrm{C} 00334$ \\
\hline Linoleic acid & 9.48 & 280.4455 & $\mathrm{C}_{18} \mathrm{H}_{32} \mathrm{O}_{2}$ & C01595 \\
\hline L-valine & 1.02 & 117.1463 & $\mathrm{C}_{5} \mathrm{H}_{11} \mathrm{NO}_{2}$ & $\mathrm{C} 00183$ \\
\hline Creatine & 1.02 & 131.1332 & $\mathrm{C}_{4} \mathrm{H}_{9} \mathrm{~N}_{3} \mathrm{O}_{2}$ & $\mathrm{C} 00300$ \\
\hline Cholesterol sulfate & 8.43 & 466.717 & $\mathrm{C}_{27} \mathrm{H}_{46} \mathrm{O}_{4} \mathrm{~S}$ & C18043 \\
\hline Arachidonic acid & 9.16 & 304.4669 & $\mathrm{C}_{20} \mathrm{H}_{32} \mathrm{O}_{2}$ & C00219 \\
\hline L-tyrosine & 7.4 & 181.1885 & $\mathrm{C}_{9} \mathrm{H}_{11} \mathrm{NO}_{3}$ & C00082 \\
\hline Palmitoleic acid & 9.5 & 254.4082 & $\mathrm{C}_{16} \mathrm{H}_{30} \mathrm{O}_{2}$ & $\mathrm{C} 08362$ \\
\hline Alpha-linolenic acid & 9.44 & 278.4296 & $\mathrm{C}_{18} \mathrm{H}_{30} \mathrm{O}_{2}$ & $\mathrm{C} 06427$ \\
\hline Oleic acid & 9.55 & 282.4614 & $\mathrm{C}_{18} \mathrm{H}_{34} \mathrm{O}_{2}$ & $\mathrm{C} 00712$ \\
\hline Biotin & 3.61 & 244.311 & $\mathrm{C}_{10} \mathrm{H}_{16} \mathrm{~N}_{2} \mathrm{O}_{3} \mathrm{~S}$ & $\mathrm{C} 00120$ \\
\hline L-proline & 0.99 & 115.1305 & $\mathrm{C}_{5} \mathrm{H}_{9} \mathrm{NO}_{2}$ & C00148 \\
\hline L-leucine & 1.35 & 131.1729 & $\mathrm{C}_{6} \mathrm{H}_{13} \mathrm{NO}_{2}$ & C00123 \\
\hline Glycine & 3.72 & 75.0666 & $\mathrm{C}_{2} \mathrm{H}_{5} \mathrm{NO}_{2}$ & $\mathrm{C} 00037$ \\
\hline D-glucose & 0.99 & 180.1559 & $\mathrm{C}_{6} \mathrm{H}_{12} \mathrm{O}_{6}$ & $\mathrm{C} 00031$ \\
\hline L-Serine & 14.76 & 105.0926 & $\mathrm{C}_{3} \mathrm{H}_{7} \mathrm{NO}_{3}$ & $\mathrm{C} 00065$ \\
\hline 4-Hydroxyphenylacetic acid & 1.95 & 152.1473 & $\mathrm{C}_{8} \mathrm{H}_{8} \mathrm{O}_{3}$ & $\mathrm{C} 00642$ \\
\hline
\end{tabular}

TABLE 3: The average changes of potential biomarkers (VIP >1) in the urine.

\begin{tabular}{|c|c|c|c|}
\hline Metabolite & Control & Model & HQT \\
\hline m-Methylhippuric acid & $96.02 \pm 14.06$ & $171.94 \pm 87.40^{*} \uparrow$ & $131.78 \pm 65.45 \downarrow$ \\
\hline 5-Hydroxyindoleacetic acid & $1.64 \pm 0.36$ & $3.30 \pm 2.18^{*} \uparrow$ & $1.51 \pm 0.67^{\#} \downarrow$ \\
\hline 1,2,3-Propanetricarboxylic acid & $3.49 \pm 0.58$ & $4.48 \pm 0.91^{*} \uparrow$ & $4.33 \pm 0.74 \downarrow$ \\
\hline Ethanolamine & $1.51 \pm 0.39$ & $2.03 \pm 0.52^{*} \uparrow$ & $1.60 \pm 0.29^{\#} \downarrow$ \\
\hline Adipic acid & $2.56 \pm 0.23$ & $2.92 \pm 0.39 * \uparrow$ & $4.83 \pm 1.91$ \\
\hline L-isoleucine & $9.30 \pm 1.92$ & $11.27 \pm 1.75^{*} \uparrow$ & $11.79 \pm 0.31$ \\
\hline Pyroglutamic acid & $5.20 \pm 1.43$ & $7.98 \pm 2.68^{*} \uparrow$ & $7.34 \pm 2.28 \downarrow$ \\
\hline L-histidine & $0.16 \pm 0.05$ & $0.25 \pm 0.07^{* * \uparrow}$ & $0.16 \pm 0.10^{\#} \downarrow$ \\
\hline 4-Hydroxyproline & $0.68 \pm 0.11$ & $0.96 \pm 0.25^{* *} \uparrow$ & $1.38 \pm 0.33$ \\
\hline Sarcosine & $0.32 \pm 0.04$ & $0.20 \pm 0.07^{* *} \downarrow$ & $0.52 \pm 0.16^{\# \# \uparrow}$ \\
\hline 3-Methylhistidine & $0.75 \pm 0.11$ & $1.01 \pm 0.30^{*} \uparrow$ & $1.57 \pm 0.54$ \\
\hline Gamma-aminobutyric acid & $33.97 \pm 6.74$ & $46.78 \pm 12.31^{*} \uparrow$ & $32.65 \pm 14.94^{\#} \downarrow$ \\
\hline Linoleic acid & $5.57 \pm 1.26$ & $4.45 \pm 0.42^{*} \downarrow$ & $6.54 \pm 2.66^{\#} \uparrow$ \\
\hline L-valine & $40.17 \pm 11.32$ & $82.31 \pm 42.31^{*} \uparrow$ & $73.53 \pm 28.53 \downarrow$ \\
\hline Creatine & $7.04 \pm 1.94$ & $5.06 \pm 0.99^{*} \downarrow$ & $6.05 \pm 0.98^{\#} \uparrow$ \\
\hline Cholesterol sulfate & $0.15 \pm 0.02$ & $0.24 \pm 0.07^{* *} \uparrow$ & $0.22 \pm 0.09 \downarrow$ \\
\hline Arachidonic acid & $0.57 \pm 0.17$ & $0.39 \pm 0.24^{*} \downarrow$ & $0.60 \pm 0.18^{\#} \uparrow$ \\
\hline L-tyrosine & $0.69 \pm 0.12$ & $0.41 \pm 0.17^{* *} \downarrow$ & $0.68 \pm 0.25^{\#} \uparrow$ \\
\hline Palmitoleic acid & $5.35 \pm 0.82$ & $4.47 \pm 0.78^{*} \downarrow$ & $6.36 \pm 2.23^{\#} \uparrow$ \\
\hline Alpha-linolenic acid & $5.80 \pm 1.05$ & $4.64 \pm 0.55^{*} \downarrow$ & $6.54 \pm 2.66 \uparrow$ \\
\hline Oleic acid & $6.87 \pm 1.68$ & $5.06 \pm 1.24^{*} \downarrow$ & $6.73 \pm 1.99^{\#} \uparrow$ \\
\hline Biotin & $1.44 \pm 0.05$ & $1.85 \pm 0.13^{* *} \uparrow$ & $2.27 \pm 1.01$ \\
\hline L-proline & $0.14 \pm 0.04$ & $0.25 \pm 0.03^{* *} \uparrow$ & $0.35 \pm 0.02$ \\
\hline L-leucine & $0.56 \pm 0.07$ & $0.71 \pm 0.12^{* *} \uparrow$ & $0.65 \pm 0.21 \downarrow$ \\
\hline Glycine & $3.67 \pm 0.34$ & $5.83 \pm 2.97^{*} \uparrow$ & $4.38 \pm 2.45 \downarrow$ \\
\hline D-glucose & $3.87 \pm 1.02$ & $7.77 \pm 7.72 * \uparrow$ & $6.28 \pm 2.30 \downarrow$ \\
\hline L-serine & $11.85 \pm 4.54$ & $6.21 \pm 2.04^{* *} \downarrow$ & $9.01 \pm 5.01 \uparrow$ \\
\hline 4-Hydroxyphenylacetic acid & $23.13 \pm 1.82$ & $33.91 \pm 6.71^{* *} \uparrow$ & $31.50 \pm 4.56 \downarrow$ \\
\hline
\end{tabular}

${ }^{*} P<0.05,{ }^{* *} P<0.01$ vs normal control; ${ }^{\#} P<0.05$, \#\# $P<0.01$ vs model group. Arrows indicate upregulated $(\uparrow)$ or downregulated $(\downarrow)$ metabolites in the model group compared with the control group and HQT group compared with model group. 


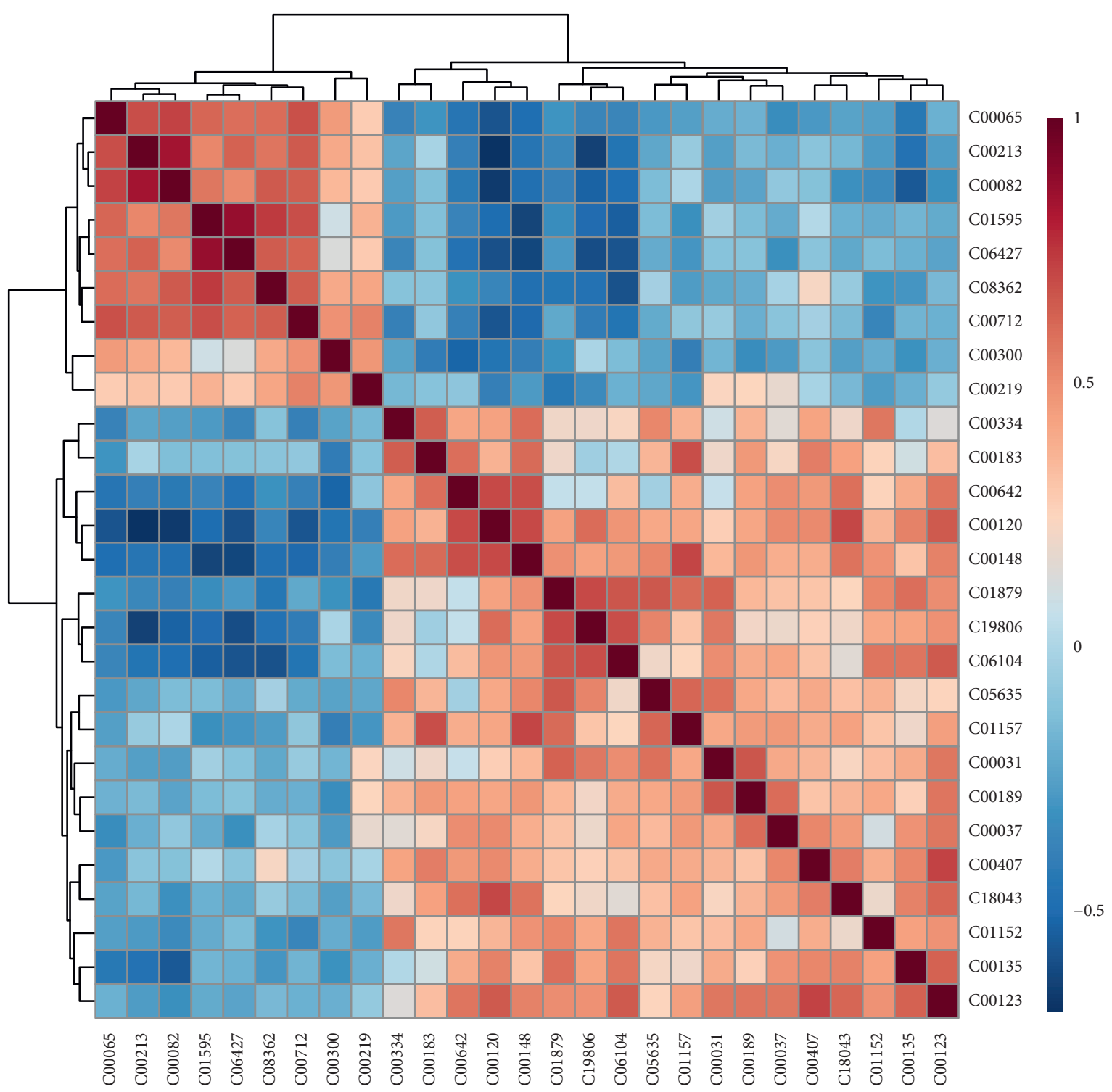

FIGURE 5: The correlation analysis of potential molecular markers in the UC model compared to the normal group.

opposite. As shown in Figure 5, amino acids and organic acids accumulate in an elevated trend in the model group, while fatty acids accumulate in a decreasing trend.

3.9. Exploratory Analysis of Diversified ROC Curves. Screening for the clinical diagnosis of potential urinary biomarkers metabolomics is an important task, and the use of ROC curves to evaluate the diagnostic accuracy of biomarkers has been successful in many studies $[25,26]$. AUC between 0.9 and 0.7 represents the diagnostic accuracy of a biomarker. Biomarkers with AUC values greater than 0.9 are highly accurate. As shown in Figure 6, the AUC value is $>0.9$, and the diagnostic sensitivity and specificity are high, indicating that metabolites used as markers for disease diagnosis have high clinical diagnostic value.

3.10. Metabolic Pathways Analysis. A total of 8 metabolic pathways $(p$ value $<0.01)$ related to UC impacted in the urine. These pathways included valine, leucine, and isoleucine biosynthesis, biosynthesis of unsaturated fatty acids, arginine and proline metabolism, and $\mathrm{ABC}$ transporters (Table 4). The abovementioned pathways may be the latent mechanisms underlying the changes of endogenous substance metabolism after 10 days of HQT exposure. Furthermore, several major metabolic disorders are connected in series, showing that UC is a disease involving multiple metabolic disorders (Figures 7 and 8).

\section{Discussion}

This study attempts to clarify the complex pathophysiological process from a metabolic perspective with the UPLC-QExactive Orbitrap MS high-sensitivity detection platform. The monitoring of these differential metabolites is helpful to further understand the mechanism of action of HQT on UC. The following is a discussion of the biological significance of the molecular markers of the abovementioned potential molecules. 


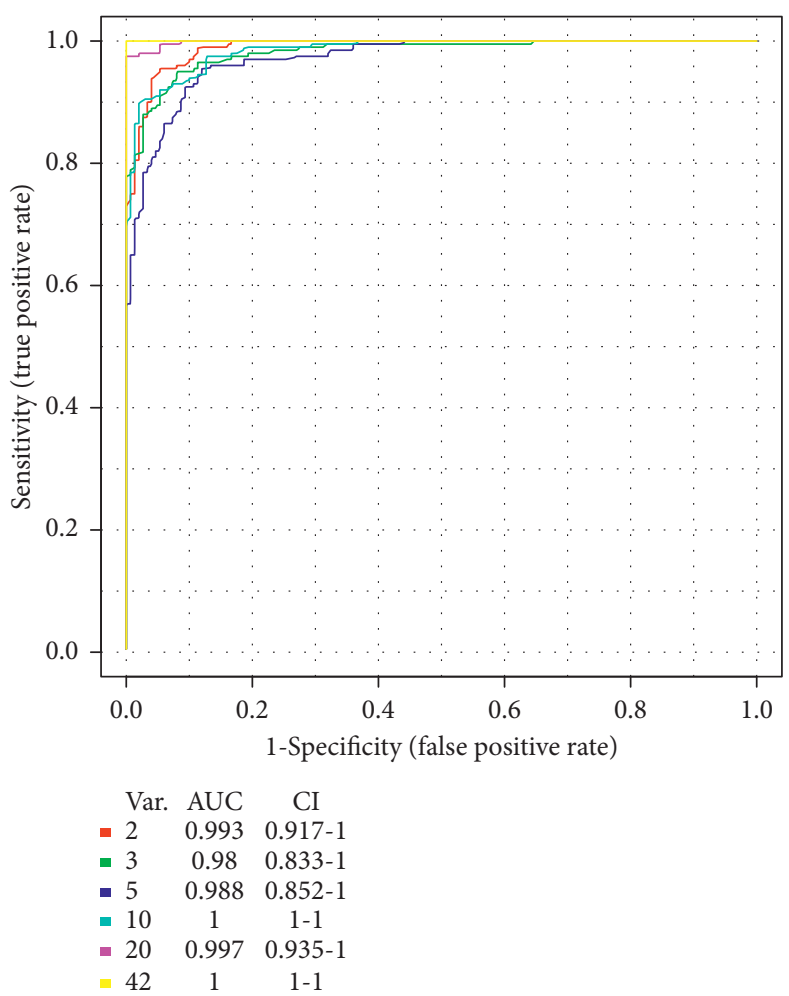

(a)

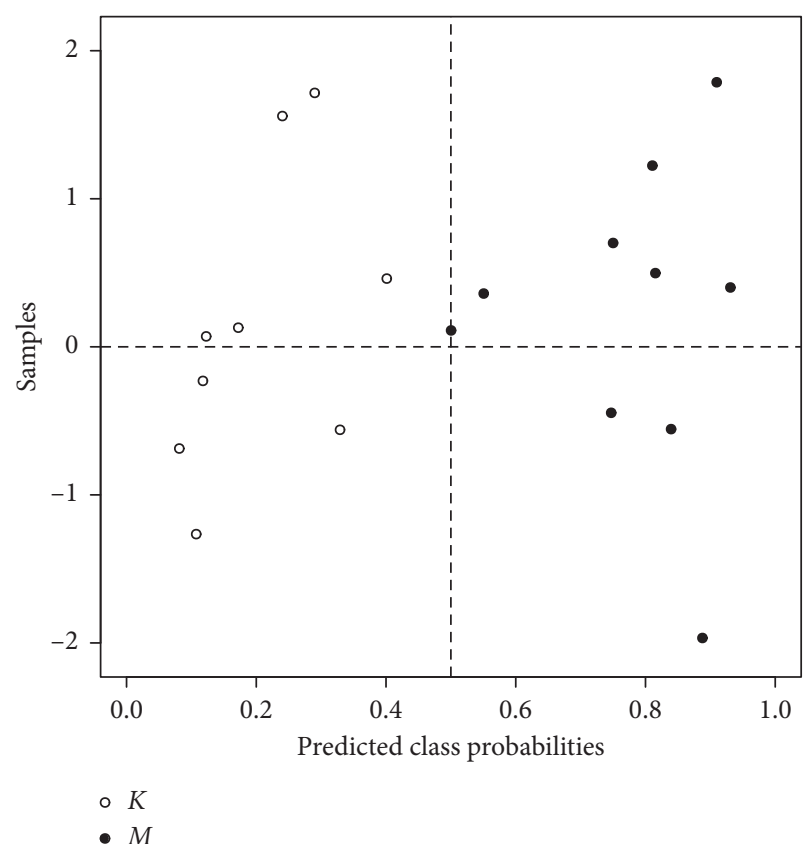

(b)

FIgURE 6: Comparison of the control and model groups of urine samples from multiple ROC curve analysis.

TABLE 4: Result for urine pathway analysis with MBRole.

\begin{tabular}{lccc}
\hline Pathway & Value & In set & $\%$ \\
\hline Aminoacyl-tRNA biosynthesis & 0.0000 & 8 & 32 \\
ABC transporters & 0.0000 & 8 & 32 \\
Arginine and proline metabolism & 0.0000 & 5 & 20 \\
Glycine, serine, and threonine metabolism & 0.0000 & 4 & 16 \\
Biosynthesis of unsaturated fatty acids & 0.0011 & 4 & 16 \\
Valine, leucine, and isoleucine biosynthesis & 0.0017 & 3 & 12 \\
Cyanoamino acid metabolism & 0.0051 & 3 & 12 \\
Valine, leucine, and isoleucine degradation & 0.0051 & 3 & 12 \\
\hline
\end{tabular}

4.1. Effects of HQT Ton the Amino Acid Metabolism in UC Rats. Amino acid is the basic functional unit that constitutes protein molecules and is one of the essential nutrients for the human body. In the UC model group, the amino acid content was generally increased. Some scholars suggested that the body can repair the damaged mucosa by decomposing proteins, causing amino acid accumulation [27]. Leucine and isoleucine promote the body's anabolism (muscle growth) in a special way, relieve muscle breakdown, and play an important role in the repair of skeletal muscle microdamage [28]. Chinese herbalists believe that diarrhea manifests itself as the loss of transportation, lack of subtle substances, no muscle growth, fatigue, weight loss, etc. These symptoms are associated with excessive loss of isoleucine, valine, and leucine in the urine, which may lead to lack of nutrition.

Recently, studies have suggested that histidine and glycine have antioxidation and anti-inflammatory effects.
They have been used in the treatment of diseases such as peptic ulcer, anemia, and allergies. For the treatment of inflammation of the gastrointestinal tract, histidine can exert anti-inflammatory functions and remove toxins, thereby enhancing the body's antioxidant capacity [29].

Gamma-aminobutyric acid (GABA), an active amino acid, is an important inhibitory neurotransmitter in the gastrointestinal nervous system [30]. GABA regulates the central nervous system, promotes cerebral cortical function and autonomic nerve function recovery, and enhances gastrointestinal motility [31]. GABA content in the model group was significantly increased, indicating abnormalities in the gastrointestinal nervous system of the rats. This may also be the main cause of gastrointestinal dysfunction of UC rats. After the administration of HQT, the GABA content was significantly increased. HQT may improve gastrointestinal tract sensitivity by regulating GABA, thereby improving diarrhea.

\subsection{Effects of HQT on the Fatty Acid Synthesis and FABPs in} $U C$ Rat. Linoleic acid is a functional polyunsaturated fatty acid that enhances the body's immune function. It is mainly used to increase the production of nonspecific antibodies, promote lymphocyte proliferation, and enhance the phagocytic ability of phagocytic cells. Linoleic acid can increase the secretion of IgG, IgA, and IgM from rat intestinal lymph nodes and spleen lymphocytes, reducing the release of inflammatory cytokines [32]. The results of this study showed that the level of linoleic acid in the urine of the 


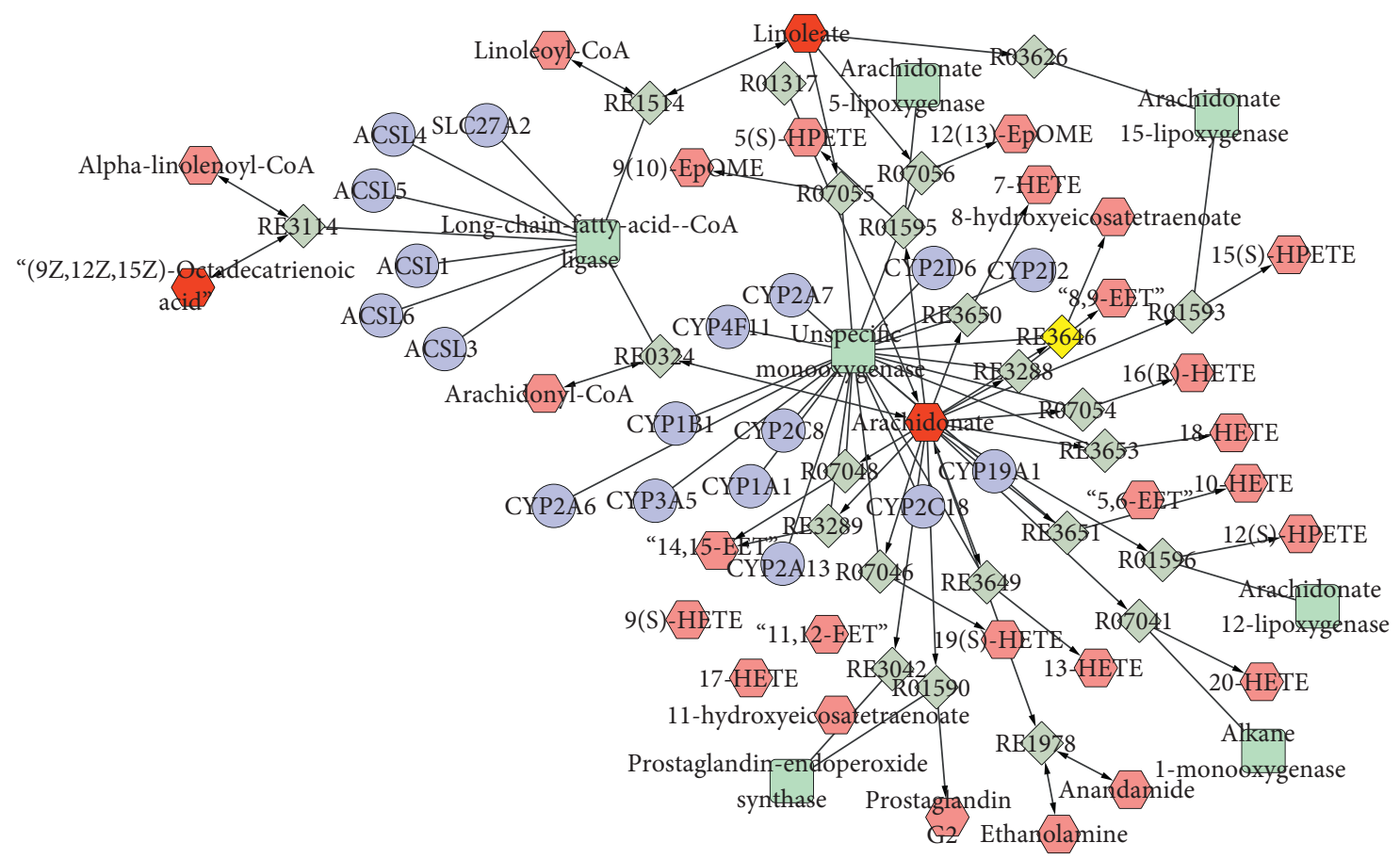

FIGURE 7: Compound-reaction-enzyme-gene network analysis of major endogenous metabolites in testis.

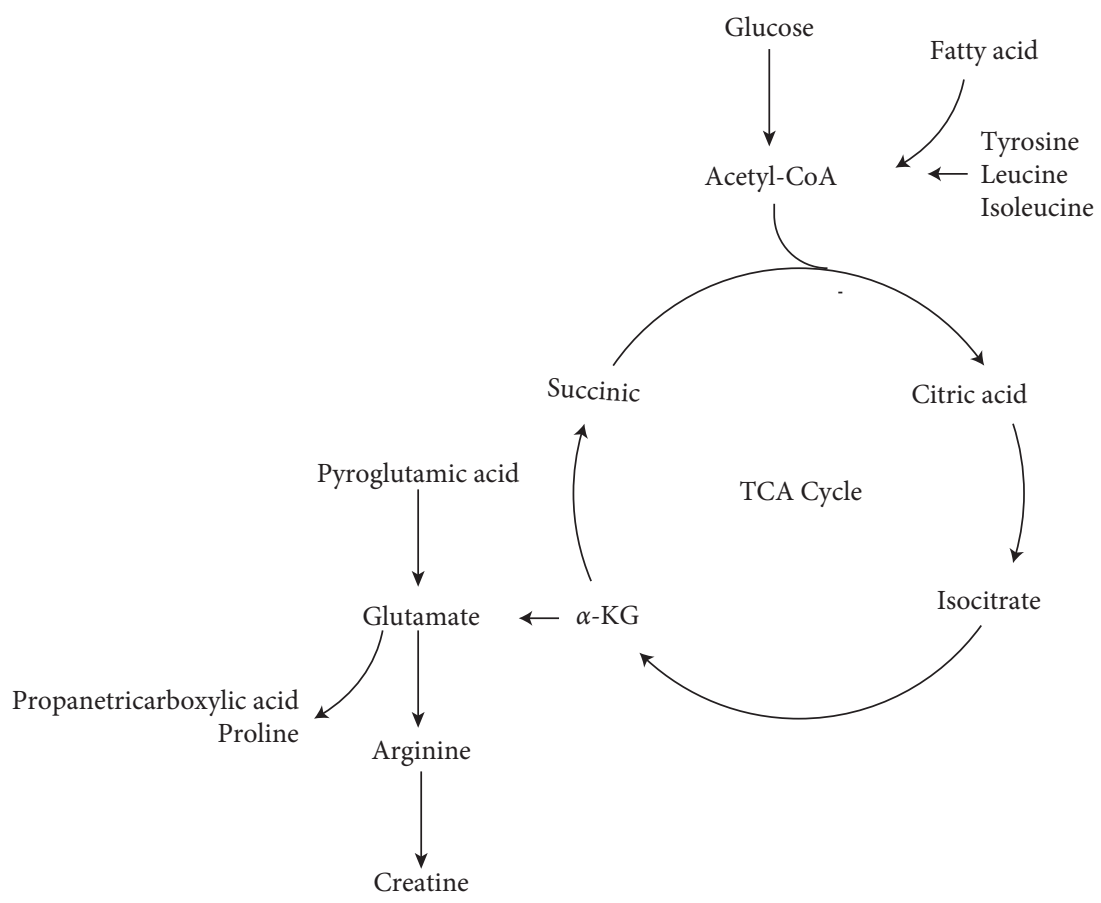

Figure 8: Metabolic profiles of potentially different metabolites in urine.

model group was significantly decreased, and the levels of inflammatory factors IL-17, $\mathrm{PGE}_{2}$, and $\mathrm{NO}$ in serum were increased, suggesting that the increase of inflammatory factors may be related to the decrease of linoleic acid level.

Simultaneously, Figure 7 displays that arachidonic acid levels were highly correlated with long-chain acyl-CoA ligase expression. The decreasing arachidonic acid may influence fatty acid synthesis, consistent with the metabolic pathway analysis. In the urine, the levels of linoleic acid, $\alpha$-linolenic acid, and oleic acid, closely related to the activity of longchain fatty acyl-CoA synthetase, also showed decreasing trends. The result demonstrated that fatty acid synthesis was disturbed by UC. In addition, the transport of fatty acids is believed to be performed by the FABP family, and FABPs activation can increase uptake of fatty acids [33]. The result showed that FABP1 and FABP2 were downregulated in the 
inflamed UC mucosa. It indicates that the genes of FABP1 and FABP2 were inhibited, affecting fatty acid uptake. This also confirms the metabolic pathway of $\mathrm{ABC}$ transporters. After 10 days of exposure to HQT, arachidonic acid, linoleic acid, $\alpha$-linolenic acid, oleic acid, FABP1, and FABP2 were upregulated, indicating HQT profoundly regulates the disrupted fatty acid synthesis and metabolism.

\subsection{Effects of HQTon the TCA Cycle and Energy Supply in UC} Rat. Glucose, pyruvic acid, and fumaric acid are important endogenous substances for the TCA cycle. The proportion of pyruvic acid and fumaric acid was lower than that in the normal rat, and the proportion of glucose and lactic acid were higher than that in the control rat (Tables 3 and 5). The results illustrated that it might have been caused by the impaired TCA cycle. The increasing trend of lactic acid in UC may validate the hypothesis that the rat obtains energy from anaerobic glycolysis and thus produces lactic acid even under aerobic conditions [34].

Creatine provides energy to muscles and nerve cells. It is a nitrogen-containing organic acid that naturally occurs in vertebrates [35]. Due to the small amount of ATP stored in the body, the existing ATP will soon be consumed during exercise. Creatine can quickly synthesize ATP to provide energy to meet the body's movements [36]. Roediger [37] believes that UC is an energy-deficiency disease. A large amount of ATP was depleted in the UC rat, causing creatine to rapidly synthesize ATP to provide energy, which may be the main reason for the decline of creatine level in UC rats. The observed decreased creatine level was also another manifestation of the insufficient ATP supply.

This study explored the mechanism of action of HQT in rats with UC from the perspective of metabolomics. It was found to act by regulating multiple metabolic pathways such as valine, leucine, and isoleucine biosynthesis, biosynthesis of unsaturated fatty acids, arginine and proline metabolism, and $\mathrm{ABC}$ transporters. It embodies the characteristics of multitarget and synergy of traditional Chinese medicine compounds. In addition to amino acids, lipids and other ingredients, cholesterol sulfate, 2-methyl hippuric acid, 4hydroxyphenylacetic acid, and other ingredients will also affect the progression of UC.

The UC biomarkers obtained in this experiment have something in common with the clinic $[38,39]$. Arginine and proline metabolism and glycine, serine, and threonine metabolism are their common pathways, which proves that the development of UC is related to the abovementioned metabolic pathways. In addition, HQT has different degrees of regulation on the abovementioned biomarkers. These biomarkers are mainly amino acids, neurotransmitters, and fatty acids. These biomarkers are similar to those associated with UC in humans. Therefore, we speculate that HQT may work by regulating the abovementioned metabolic pathways. The speculation for this part also needs to be verified in conjunction with target metabolomics studies. Secondly, proteomics, transcriptomics, and other biological experimental methods are used to explore the biological significance of these biomarkers in detail.
TABLE 5: Differential endogenous metabolites of UC rats.

\begin{tabular}{lccc}
\hline Metabolite & Control & Model & HQT \\
\hline Fumaric acid & $6.75 \pm 1.43$ & $5.98 \pm 0.93^{*} \downarrow$ & $6.93 \pm 2.12 \uparrow$ \\
Pyruvic acid & $7.34 \pm 1.46$ & $6.06 \pm 0.74^{*} \downarrow$ & $7.17 \pm 2.21 \uparrow$ \\
Lactic acid & $16.11 \pm 1.60$ & $18.49 \pm 2.78^{*} \uparrow$ & $11.30 \pm 1.43^{\#} \uparrow$ \\
\hline${ }^{*} P<0.05,{ }^{* *} P<0.01$ vs normal control; ${ }^{\#} P<0.05,{ }^{\#} P<0.01$ vs model \\
group. Arrows indicate upregulated $(\uparrow)$ or downregulated $(\downarrow)$ metabolites in \\
the model group compared with the control group and HQT group \\
compared with model group.
\end{tabular}

\section{Data Availability}

The datasets used and analyzed during the current study are available from the corresponding author upon reasonable request.

\section{Conflicts of Interest}

The authors declare no conflicts of interest.

\section{Authors' Contributions}

Dunfang Wang and Xuran Ma contributed equally to this work.

\section{Acknowledgments}

The work was supported by the National Natural Science Foundation of China (nos. 81273662 and 81473592) and the Fundamental Research Funds for the Central Public Welfare Research Institutes (zxkt17029 and zxkt18016).

\section{References}

[1] B. Wang, X. D. Zhu, Y. Q. Duan et al., "Effect of paeoniflorin on colon tissue pathological morphology and the balance of anti-inflammatory and proinflammatory factors in rats with ulcerative colitis," Chinese Journal of Integrated Traditional \& Western Medicine on Digestion, vol. 21, pp. 1-4, 2013.

[2] H. F. Hammer, "Gut microbiota and inflammatory bowel disease," Digestive Diseases, vol. 29, no. 6, pp. 550-553, 2011.

[3] R. Cooney and D. Jewell, "The genetic basis of inflammatory bowel disease," Digestive Diseases, vol. 27, no. 4, pp. 428-442, 2009.

[4] V. Leone, E. B. Chang, and S. Devkota, "Diet, microbes, and host genetics: the perfect storm in inflammatory bowel diseases," Journal of Gastroenterology, vol. 48, no. 3, pp. 315-321, 2013.

[5] M. Wang, L. Chen, D. Liu et al., "Metabolomics highlights pharmacological bioactivity and biochemical mechanism of traditional Chinese medicine," Chemico-Biological Interactions, vol. 273, pp. 133-141, 2017.

[6] Y. Jia, Q. Guan, Y. Jiang et al., "Amelioration of dextran sulphate sodium-induced colitis in mice by echinacosideenriched extract of Cistanche tubulosa," Phytotherapy Research, vol. 28, no. 1, pp. 110-119, 2014.

[7] D. F. Wang, Y. L. Wang, Y. W. Wang et al., "Effect of Huangqin Tang on the function of regulatory TLR4/MyD88 signal pathway in rats with ulcerative colitis," Acta Pharmaceutica Sinica, vol. 51, no. 10, pp. 1558-1563, 2016.

[8] Y. W. Wang, H. H. Zhang, Y. L. Wang et al., "Effect of Huangqin Tang on the regulatory NF- $\kappa$ B p65 signal pathway 
in rats with ulcerative colitis," Acta Pharmaceutica Sinica, no. 1, pp. 21-27, 2015.

[9] J. R. Idle and F. J. Gonzalez, "Metabolomics," Cell Metabolism, vol. 6, no. 5, pp. 348-351, 2007.

[10] J. K. Nicholson and I. D. Wilson, "Understanding "global" systems biology: metabonomics and the continuum of metabolism," Nature Reviews Drug Discovery, vol. 2, no. 8, pp. 668-676, 2003.

[11] D.-Q. Chen, H. Chen, L. Chen et al., "Metabolomic application in toxicity evaluation and toxicological biomarker identification of natural product," Chemico-Biological Interactions, vol. 252, pp. 114-130, 2016.

[12] H. Chen, H. Miao, Y.-L. Feng et al., "Metabolomics in dyslipidemia," Advances in Clinical Chemistry, Elsevier, vol. 66, pp. 101-119, , Amsterdam, Netherlands, 2014.

[13] J. K. Nicholson, "Global systems biology, personalized medicine and molecular epidemiology," Molecular Systems Biology, vol. 2, no. 1, p. 52, 2006.

[14] C. Legido-Quigley, L. McDermott, H. Vilca-Melendez et al., "Bile UPLC-MS fingerprinting and bile acid fluxes during human liver transplantation," Electrophoresis, vol. 32, no. 15, pp. 2063-2070, 2011.

[15] Z. An, Y. Chen, R. Zhang et al., "Integrated ionization approach for RRLC-MS/MS-based metabonomics: finding potential biomarkers for lung cancer," Journal of Proteome Research, vol. 9, no. 8, pp. 4071-4081, 2010.

[16] I. R. Lanza, S. Zhang, L. E. Ward et al., "Quantitative metabolomics by $1 \mathrm{H}-\mathrm{NMR}$ and LC-MS/MS confirms altered metabolic pathways in diabetes," PLoS One, vol. 5, no. 5, Article ID e10538, 2010.

[17] Y.-Y. Zhao, X.-L. Cheng, Vaziri et al., "UPLC-based metabonomic applications for discovering biomarkers of diseases in clinical chemistry," Clinical Biochemistry, vol. 47, no. 15, pp. 16-26, 2014.

[18] G. P. Morris, P. L. Beck, M. S. Herridge et al., "Hapten-induced model of chronic inflammation and ulceration in the rat colon," Gastroenterology, vol. 96, no. 3, pp. 795-803, 1989.

[19] M. Ballester, A. Castelló, E. Ibáñez et al., "Real-time quantitative PCR-based system for determining transgene copy number in transgenic animals," Biotechniques, vol. 37 , no. 4, pp. 610-613, 2004.

[20] A. Makarov, "Electrostatic axially harmonic orbital trapping: a high-performance technique of mass analysis," Analytical Chemistry, vol. 72, no. 6, pp. 1156-1162, 2000.

[21] D. S. Wishart, Y. D. Feunang, A. Marcu et al., "HMDB 4.0: the human metabolome database for 2018," Nucleic Acids Research, vol. 46, no. D1, pp. D608-D617, 2017.

[22] M. Kanehisa, S. Goto, S. Kawashima et al., "KEGG: kyoto encyclopedia of genes and genomes," Nucleic Acids Research, vol. 28 , no. 1 , pp. $27-30,2000$.

[23] M. Kanehisa, M. Araki, S. Hattori et al., "KEGG for linking genomes to life and the environment," Nucleic Acids Research, vol. 36, pp. D480-D484, 2007.

[24] T. Li, S. Zhuang, Y. Wang et al., "Flavonoid profiling of a traditional Chinese medicine formula of Huangqin Tang using high performance liquid chromatography," Acta Pharmaceutica Sinica B, vol. 6, no. 2, pp. 148-157, 2016.

[25] H. Wu, R. Xue, Z. Tang et al., "Metabolomic investigation of gastric cancer tissue using gas chromatography/mass spectrometry," Analytical and Bioanalytical Chemistry, vol. 396, no. 4, pp. 1385-1395, 2010.

[26] Y. Li, X. Song, and X. Zhao, "Serum metabolic profiling study of lung cancer using ultra high performance liquid chromatography/quadrupole time of flight mass spectrometry," Journal of Chromatography B, vol. 966, pp. 47-53, 2014.

[27] V De Preter, K Machiels, M. Joossens et al., "Faecal metabolite profiling identifies medium-chain fatty acids as discriminating compounds in IBD," Gut, vol. 64, no. 3, pp. 447-458, 2015.

[28] K. C. J. Yuen, B. M. K. Biller, M. E. Molitch et al., "Is lack of recombinant growth hormone $(\mathrm{GH})$-releasing hormone in the United States a setback or time to consider glucagon testing for adult GH deficiency?" The Journal of Clinical Endocrinology \& Metabolism, vol. 94, no. 8, pp. 2702-2707, 2009.

[29] A. M. Wade and H. N. Tucker, "Antioxidant characteristics of L-histidine 1," Journal of Nutritional Biochemistry, vol. 9, no. 6, pp. 308-315, 1998.

[30] S. Y. Yang and Z. Lu, "Research progress on microbial glutamate decarboxylase," Food Science, vol. 9, pp. 528-533, 2005.

[31] K. Zhang, L. Ren, D. Li et al., "Influence of metabonomics of liver depression functional dyspepsia rats on electric acupuncture," Journal of Liaoning University Traditional Chinese Medicine, vol. 6, pp. 110-114, 2016.

[32] E. C. Laiakis, G. A. J. Morris, A. J. Fornace et al., "Metabolomic analysis in severe childhood pneumonia in the Gambia, West Africa: findings from a pilot study," PLoS One, vol. 5, no. 9, Article ID e12655, 2010.

[33] M. M. A. L. Pelsers, Z. Namiot, W. Kisielewski et al., "Intestinal-type and liver-type fatty acid-binding protein in the intestine. Tissue distribution and clinical utility," Clinical Biochemistry, vol. 36, no. 7, pp. 529-535, 2003.

[34] T. H. Hereng, K. B. P. Elgstøen, F. H. Cederkvist et al., "Exogenous pyruvate accelerates glycolysis and promotes capacitation in human spermatozoa," Human Reproduction, vol. 26, no. 12, pp. 3249-3263, 2011.

[35] X. Xiao-xiang, Y. Cao, K.-y. Fu et al., "The changes of energy metabolites in rat muscle were caused by occlusal interference," Journal of Peking University, vol. 49, no. 1, pp. 25-30, 2017.

[36] D.-q. Zhou, L. Gong, and Z.-y. Qian, "The physiological function and motion of creatine kinase," Journal of Anqing Normal University, vol. 22, no. 3, pp. 113-118, 2016.

[37] W. E. W. Roediger, "The colonic epithelium in ulcerative colitis: an energy-deficiency disease?" The Lancet, vol. 316, no. 8197 , pp. $712-715,1980$.

[38] J. Wang, Y. Cao, Z. Li et al., "Plasma metabolomic study on deficiency or excessiveness patterns of chronic recurrent ulcerative colitis basedon H-nuclear magnetic resonance," Journal of Beijing University of Traditional Ch5inese Medicine, vol. 41, no. 9, pp. 85-90, 2018.

[39] L. Xin, Ulcerative Colitis Amino Acid Metabolic Profile Analysis and Its Clinical Significance, Shanxi Medical University, Taiyuan, China, 2014. 